\title{
IncRNA LINC00473 promotes proliferation, migration, invasion and inhibition of apoptosis of non-small cell lung cancer cells by acting as a sponge of miR-497-5p
}

\author{
SHAO-HUA XU ${ }^{1}$, YU-HONG BO ${ }^{2}$, HAN-CHEN MA ${ }^{1}$, HUA-NAN ZHANG ${ }^{1}$ and MING-JU SHAO ${ }^{3}$ \\ ${ }^{1}$ Department of Respiration, The Second Hospital of Shandong University, Ji'nan, Shandong, 250000; \\ ${ }^{2}$ Department of Respiration, Weihai Municipal Hospital, Weihai, Shandong 264200; \\ ${ }^{3}$ Department of Emergency Medicine, The Second Hospital of Shandong University, Ji'nan, Shandong 250000, P.R. China
}

Received January 17, 2020; Accepted January 14, 2021

DOI: $10.3892 /$ ol.2021.12690

\begin{abstract}
Lung cancer is the leading cause of cancer-associated death worldwide and exhibits a poor prognosis. The present study aimed to determine the effect of long non-coding (lnc)RNA-LINC00473 on the development of non-small cell lung cancer (NSCLC) cells by regulating the expression of microRNA (miR)-497-5p. Reverse transcription-quantitative PCR was conducted to detect the level of LINC00473 and miR-497-5p. An MTT assay, flow cytometry and Transwell tests were performed to evaluate the proliferation, apoptosis, migration and invasion of NSCLC cells. Western blotting was performed to detect the expression of apoptosis- and migration-related proteins. RNA immunoprecipitation and a luciferase reporter assay were performed to verify the regulatory relationship between lncRNA-LINC00473 and miR-497-5p. LINC00473 expression was upregulated in lung cancer tissues and NSCLC cells (A549 and H1299) when compared with adjacent tissues or human bronchial epithelial cell lines and the 5-year survival rate was lower in patients with high LINC00473 expression compared with in patients with low LINC00473 expression. A negative correlation between LINC00473 and miR-497-5p was observed in lung cancer tissues. Proliferation, migration and invasion as well as the related protein levels were increased in A549 and H1299 transfected with pcDNA3.1-LINC00473, while the opposite results were obtained in A549 and H1299 transfected with small interfering (si)-LINC00473. Notably, it was demonstrated that LINC00473 could bind directly with miR-497-5p and inhibit its expression. miR-497-5p inhibitors reversed the effect of si-LINC00473. Furthermore, the present study
\end{abstract}

Correspondence to: Dr Ming-Ju Shao, Department of Emergency Medicine, The Second Hospital of Shandong University, 247 Beiyuan Street, Ji'nan, Shandong 250000, P.R. China

E-mail: shaomingju123@126.com

Key words: non-small cell lung cancer, LINC00473, miR-497-5p, proliferation, MAPK demonstrated that LINC00473 promoted the malignant behaviour of NSCLC cells via regulating the ERK/p38 and MAPK signalling pathways and the expression of miR-497-5p.

\section{Introduction}

Lung cancer is a disease with the fastest increasing morbidity and mortality rates, and is a notable public health issue (1). In 2019, there were 228,150 new cases and 142,670 deaths of lung cancer in the United States (1). It is categorised into small cell lung cancer and non-small cell lung cancer (NSCLC), with the latter composing $\sim 85 \%$ of all lung cancer cases in the United States in 2015 (2). Despite the development of therapeutic technology, the effect of lung cancer treatment remains unsatisfactory, with a low survival rate of only $15 \%(3,4)$. Therefore, it is important to elucidate the pathogenesis of lung cancer occurrence and progression.

Long non-coding (lnc)RNAs have been found to be involved in tumour initiation and tumorigenesis $(5,6)$. Furthermore, studies have shown that numerous lncRNAs, such as lncRNA cancer susceptibility 15, forkhead box protein $\mathrm{C} 2$ and JHDM1D antisense 1, promote the onset and progression of NSCLC (7-9). LINC00473 is a novel oncogenic lncRNA and is upregulated in various types of human cancer (10). Reportedly, silencing LINC00473 can block the progression of pancreatic cancer by strengthening miR-195-5p-targeted downregulation of PD-L1 (11). Niu et al (12) also reported that LINC00473 regulates the expression of MAPK1 in breast cancer by interacting with miR-198 (12). However, there have been few reports on the effects of LINC00473 in NSCLC.

microRNAs (miRNAs/miRs) are elements that can inhibit the expression of target genes by hindering mRNA translation, where imbalance can result in tumour suppressor gene dysfunction, leading to the occurrence of tumours (13). It has been reported that lncRNA can act as a sponge of miRNAs, reducing their regulatory effects on target mRNAs (14). The present study aimed to elucidate the mechanism by which LINC00473 affects the progression of NSCLC. Using bioinformatics tools, the miRNAs that bind to LINC00473 were predicted, and the miR-497-5p binding site was revealed. Previously miRNA miR-497-5p has been found to inhibit 
NSCLC cell proliferation and invasion as a tumour suppressor gene, therefore it was speculated that LINC00473 may influence the progression of NSCLC by targeting miR-497-5p and affecting its activity.

\section{Materials and methods}

Sample collection. In total, 58 NSCLC tissues and normal adjacent tissues ( $\sim 5 \mathrm{~cm}$ away from the cancerous tissues; 39 males and 19 females; age range, 29-81 years; median age, 56 years) were obtained from patients who underwent resection surgery at The Second Hospital of Shandong University (Ji'nan, China) between July 2017 and August 2018. All samples were verified after a histopathological double-blind assessment by two independent pathologists and immediately stored at $-80^{\circ} \mathrm{C}$. Five-year survival rates were calculated by the percentage of patients who survived $>60$ months after resection surgery. Each patient signed an informed consent form. The protocol of the research was approved by The Ethics Committee of The Second Hospital of Shandong University (Ji'nan, China).

Cell culture. NSCLC cell lines A549 and H1299 and normal human bronchial epithelial cell line (HBE) were obtained from the Cell Bank of the Type Culture Collection of the Chinese Academy of Sciences and cultured in RPMI-1640 medium (Gibco; Thermo Fisher Scientific, Inc.) containing 10\% bovine fetal serum (FBS), $100 \mathrm{U} / \mathrm{ml}$ penicillin and $100 \mu \mathrm{g} / \mathrm{ml}$ streptomycin sulphate (both HyClone; Cytiva) at $37^{\circ} \mathrm{C}$ in a humidified incubator with $5 \% \mathrm{CO}_{2}$ atmosphere.

Cell transfection. Once cells reached $70-80 \%$ confluence, si-LINC00473 (5 nM; 5'-GCGCCGGGAGAUGCAUCACGAU GAA-3'), pcDNA3.1-LINC00473 (Shanghai GenePharma Co., Ltd.), miR-497-5p mimics (50 nM; 5'-CAGCAGCACACUGUG GUUUGU-3') and miR-497-5p inhibitors (100 nM; 5'-ACAAA CCACAGUGUGCUGCUG-3') (Guangzhou RiboBio Co., Ltd.) were transfected into A549 and H1299 cells, respectively, using Lipofectamine 2000 (Invitrogen; Thermo Fisher Scientific, Inc.) according to the manufacturer's instructions. As a control, si-NC (5 nM; 5'-CACUGAUUUCAAAUGGUGCUAUU-3'), pcDNA3.1-NC, mimics NC (50 nM; 5'-UUCUCCGAACGU GUCACGUTT-3') and inhibitors NC (100 nM; 5'-CAGUAC UUUUGUGUAGUACAA-3') were also transfected into A549 and $\mathrm{H} 1299$ cells and were non-targeting. After $48 \mathrm{~h}$ at $37^{\circ} \mathrm{C}$, cells were collected for subsequent analyses.

Luciferase reporter assay. LINC00473-wild-type (WT) and LINC00473-mutant (MUT) inserts were ligated into pGL3 reporter vectors (Promega Corporation) and transfected into A549 and H1299 cells $\left(5 \times 10^{5}\right)$, respectively, in 24-well plates and incubated for $24 \mathrm{~h}$ at $37^{\circ} \mathrm{C}$. In parallel, miR-497-5p mimics or mimics NC LINC00473-WT or LINC00473-MUT were respectively transfected into A549 and H1299 cells using Lipofectamine 2000 according to the manufacturer's instructions. Luciferase activity was detected using the Dual-Luciferase Reporter Assay system (Promega Corporation). Renilla luciferase activity was used for normalization. Moreover, the binding site of LINC00473 and miR-497-5p was predicted using DIANA tools (http://carolina. imis.athena-innovation.gr/diana_tools/web/).
RNA immunoprecipitation. A549 and H1299 cells were lysed in complete RIP lysis buffer (EMD Millipore) and then incubated with magnetic beads (EMD Millipore) containing human anti-SNRNP70 antibody or IgG (EMD Millipore; cat. nos. CS203216 and CS200621, respectively) overnight at $4^{\circ} \mathrm{C}$. After incubation, tubes were placed on a magnetic separator (EMD Millipore) and supernatants were discarded. After washing with RIP washing buffer, the samples were subsequently incubated with proteinase $\mathrm{K}$ buffer in a heating block at $55^{\circ} \mathrm{C}$ for $30 \mathrm{~min}$. Target RNA was extracted using chloroform, purified using two salt solutions (EMD Millipore), a precipitate enhancer (EMD Millipore) and absolute ethanol, and detected by reverse transcription-quantitative (RT-q)PCR analysis.

$R T-q P C R$. Total RNA was isolated from both tissues and cells using TRIzol ${ }^{\circledR}$ reagent (Invitrogen; Thermo Fisher Scientific, Inc.) and reverse transcribed using PrimeScript ${ }^{\mathrm{TM}} \mathrm{RT}$ reagent kit (Takara Bio, Inc.) according to the manufacturer's protocol. The relative gene expression of LINC00473 and miR-497-5p was analysed using a SYBR ${ }^{\circledR}$ Premix Ex Taq ${ }^{\text {TM }}$ kit (Takara Bio, Inc.) and an ABI PRISM ${ }^{\circledR} 7500$ Two-step Sequence Detection system (Applied Biosystems; Thermo Fisher Scientific, Inc.). The PCR conditions were $95^{\circ} \mathrm{C}$ for $3 \mathrm{~min}$, followed by 40 cycles at $95^{\circ} \mathrm{C}$ for $5 \mathrm{sec}$ and $60^{\circ} \mathrm{C}$ for $30 \mathrm{sec}$. The $2^{-\Delta \Delta \mathrm{Cq}}$ method (15) was used for quantification of LINC00473 and miR-497-5p expression. GAPDH and U6 expression levels were used as reference controls for LINC00473 and miR-497-5p levels, respectively. Primers used for amplification are listed in Table I.

Colony formation assay. A total of 1,000 transfected A549 and H1299 cells were placed in 6-well plates and cultured in RPMI-1640 medium. After 14 days of continuous incubation at $37^{\circ} \mathrm{C}$, cells were stained with crystal violet for $10 \mathrm{~min}$ at room temperature (Beyotime Institute of Biotechnology) and observed manually under a light microscope (magnification, $x 40$ ) for the number of colonies to be counted ( $>50$ cells were considered as a colony).

MTT assay. The transfected cells were resuspended in RPMI-1640 with $10 \%$ FBS, seeded in a 96 -well plate $\left(5 \times 10^{3} /\right.$ well $)$, and incubated at room temperature for $24-72 \mathrm{~h}$. MTT solution was then added, followed by incubation for $4 \mathrm{~h}$ and addition of $150 \mu \mathrm{l}$ DMSO to each well. Optical density at $490 \mathrm{~nm}$ was measured using a microplate reader, and data were expressed as absorbance values.

Apoptosis analysis. Apoptosis analysis was performed using an Annexin V FITC Apoptosis kit (BD Biosciences; cat. no. 556420). Cells transfected with si-LINC00473, pcDNA3.1-LINC00473 and miR-497-5p inhibitors were cultured for $48 \mathrm{~h}$, then incubated with FITC-labelled Annexin V and PI in the dark at $25^{\circ} \mathrm{C}$ for $20 \mathrm{~min}$. Thereafter, the apoptosis rate of the cells was measured by flow cytometry using a flow cytometer (BD FACSCanto II; BD Biosciences). FlowJo version 10 software (FlowJo LLC) was used to analyse apoptosis.

Transwell migration and invasion assays. After $48 \mathrm{~h}$ of incubation, transfected A549 and H1299 cells were trypsinised and 
Table I. Primer sequences for quantitative expression analysis.

\begin{tabular}{ll}
\hline Gene & Sequence of oligonucleotides, 5'-3' \\
\hline LINC00473 & \\
Forward & \\
Reverse & GATGGAAAGGAGGGAAGG \\
microRNA-497-5p & CACAGTGGGTCCAGGGTT \\
Forward & \\
Reverse & CCTTCAGCAGCACACTGTGG \\
GAPDH & CAGTGCAGGGTCCGAGGTAT \\
Forward & \\
Reverse & AGAAGGCTGGGGCTCATTTG \\
U6 & AGGGGCCATC CACAGTCTTC \\
Forward & \\
Reverse & CTCGCTTCGGCAGCACA \\
\hline
\end{tabular}

resuspended. For invasion assays, the upper chamber surface of the Transwell insert was coated with $50 \mathrm{mg} / \mathrm{l}$ Matrigel (1:8) and air-dried at $4^{\circ} \mathrm{C}$. For both migration and invasion assays, $2 \times 10^{5}$ cells were added to the upper chamber containing serum-free RPMI-1640 medium, and the lower chamber was filled with RPMI-1640 medium supplemented with 5\% FBS. After $24 \mathrm{~h}$ of incubation, the cells were stained with crystal violet for $20 \mathrm{~min}$ at room temperature. Finally, the filters were washed with PBS and observed under a light microscope (magnification, x200). Four visual fields were randomly selected and images were captured, and the visible cells were counted.

Western blotting. After $48 \mathrm{~h}$ incubation of transfected cells, samples from each group were collected, and lysed with ice cold RIPA buffer (Sigma-Aldrich; Merck KGaA) supplemented with a protease inhibitor cocktail (Roche Diagnostics). The protein concentration was measured using a BCA kit (Takara Bio, Inc.). Proteins (10 ng/lane) were separated via $10 \%$ SDS-PAGE, followed by transfer to a PVDF membrane. After $2.5 \mathrm{~h}$ of blocking with a BSA (neoFroxx $\mathrm{GmbH}$ )-TBST solution (1x TBS, $1 \%$ Tween-20 and 5\% w/v BSA) at room temperature, the PVDF membrane was incubated with the following primary antibodies: Bax (1:1,000; cat. no. 14796), Bcl-2 (1:1,000; cat. no. 4223), Matrix metalloproteinase (MMP)-2 (1:1,000; cat. no. 40994), MMP-9 (1:1,000; cat. no. 15561), p44/42 (ERK1/2; 1:1,000; cat. no. 4695), phosphorylated-p44/42 (p-ERK1/2; Thr202/Tyr204; 1:1,000; cat. no. 4370), p38 (1:1,000; cat. no. 14451) and phosphorylated-p38 (Thr180/Tyr182; 1:1,000; cat. no. 4511) (all Cell Signalling Technology, Inc.) at $4^{\circ} \mathrm{C}$ overnight. The following day, the samples were incubated with HRP-conjugated secondary antibodies (1:2,000; cat. no. 7074; Cell Signalling Technology, Inc.) at room temperature for $2 \mathrm{~h}$. The protein blots on the membrane were imaged using a chemiluminescence kit (Pierce; Thermo Fisher Scientific, Inc.). Data were quantified using ImageJ software v1.41 (National Institutes of Health).

Statistical analysis. Data are presented as the mean \pm standard deviation of three independent experiments and were analysed using SPSS 19.0 (IBM Corp). Paired t-tests were used to determine the statistical differences between two groups. The overall survival rate was analysed by the Kaplan-Meier method and differences were tested using the log-rank test. The data difference among $>2$ groups were determined using ANOVA and Bonferroni's post-hoc test. The correlation between LINC00473 and miR-497-5p was analysed using Pearson's correlation coefficient. $\mathrm{P}<0.05$ was considered to indicate a statistically significant difference.

\section{Results}

LINC00473 expression is upregulated in NSCLC tissues and cells. RT-qPCR results indicated that LINC00473 was significantly upregulated in NSCLC tissues when compared with adjacent tissues $(\mathrm{P}<0.05$; Fig. 1A). Furthermore, survival analysis indicated that patients with a higher expression of LINC00473 had a significantly lower 5-year survival rate compared with patients with low expression of LINC00473 (log-rank P $<0.05$; Fig. 1B). Additionally, significantly increased expression of LINC00473 was observed in both A549 and H1299 cells compared with HBE cells $(\mathrm{P}<0.05$; Fig. 1C). The present results indicated that LINC00473 expression was upregulated in both NSCLC tissues and cells.

LINC00473 promotes proliferation and inhibits apoptosis of NSCLC cells. As indicated in Fig. 2A, LINC00473 was significantly upregulated in A549 and H1299 cells transfected with pcDNA3.1-LINC00473, compared with those transfected with pcDNA3.1-NC (both $\mathrm{P}<0.05$ ). Furthermore, the expression of LINC00473 in A549 and H1299 cells was significantly decreased after transfection with si-LINC00473 when compared with the si-NC group (both $\mathrm{P}<0.05$ ). The results of the MTT and colony formation assays indicated that overexpression of LINC00473 promoted proliferation, whereas its downregulation inhibited the proliferation of A549 and $\mathrm{H} 1299$ cells compared with their NC groups (all $\mathrm{P}<0.05$; Fig. 2B and C). Moreover, the apoptosis rate (Annexin $\mathrm{V}^{+} / \mathrm{PI}^{+}$ cells) significantly decreased when LINC00473 was overexpressed and significantly increased when LINC00473 was knocked down (both $\mathrm{P}<0.05$; Fig. 2D). Meanwhile, it was observed that expression levels of Bax decreased while Bcl-2 increased with LINC00473-overexpression, and the opposite was observed when LINC00473 was knocked down, with an increase in Bax and decrease in Bcl-2 expression $(\mathrm{P}<0.05$; Fig. 2E). These results suggested that LINC00473 served a promoting role in proliferation and an inhibitory role in apoptosis of NSCLC cells in vitro.

LINC00473 promotes the migration and invasion of NSCLC cells. A Transwell migration assay demonstrated that the overexpression of LINC00473 significantly enhanced the migration and invasion activities of NSCLC cells $(\mathrm{P}<0.05$; Fig. $3 \mathrm{~A}$ and $\mathrm{B})$. Conversely, migration was significantly inhibited by LINC00473-knockdown ( $\mathrm{P}<0.05$; Fig. $3 \mathrm{~A}$ and B). In addition, LINC00473-overexpression significantly increased the expression of the metastasis-associated proteins MMP-2 and MMP-9; however, LINC00473-knockdown significantly decreased their expression (all $\mathrm{P}<0.05$; Fig. 3C). The results demonstrated that LINC00473 promoted the migration and invasion of NSCLC cells in vitro. 

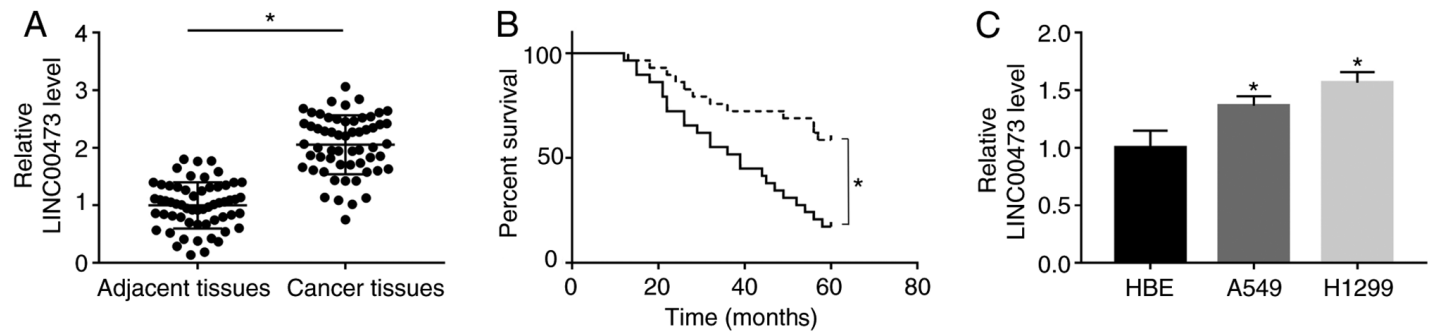

-2. Low LINC00473 expression

- High LINC00473 expression

Figure 1. LINC00473 is upregulated in lung cancer tissues, HEB, A549 and H1299 cells. (A) RT-qPCR was conducted to detect the transcription level of LINC00473. (B) Overall survival rate of patients with lung cancer with either high or low expression of LINC00473. (C) Expression of LINC00473 in human bronchial epithelial and NSCLC cell lines as quantified using RT-qPCR. RT-q, reverse transcription-quantitative.

A

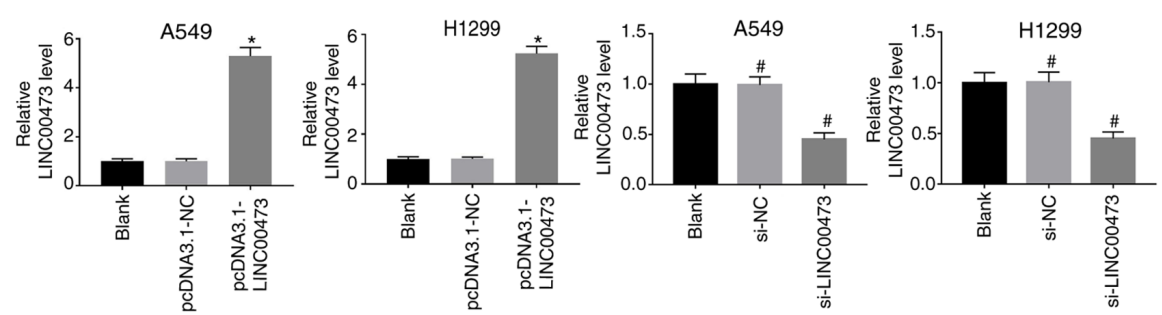

B
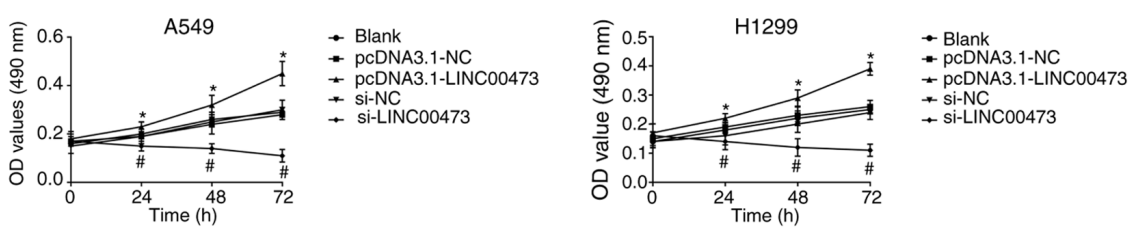

C
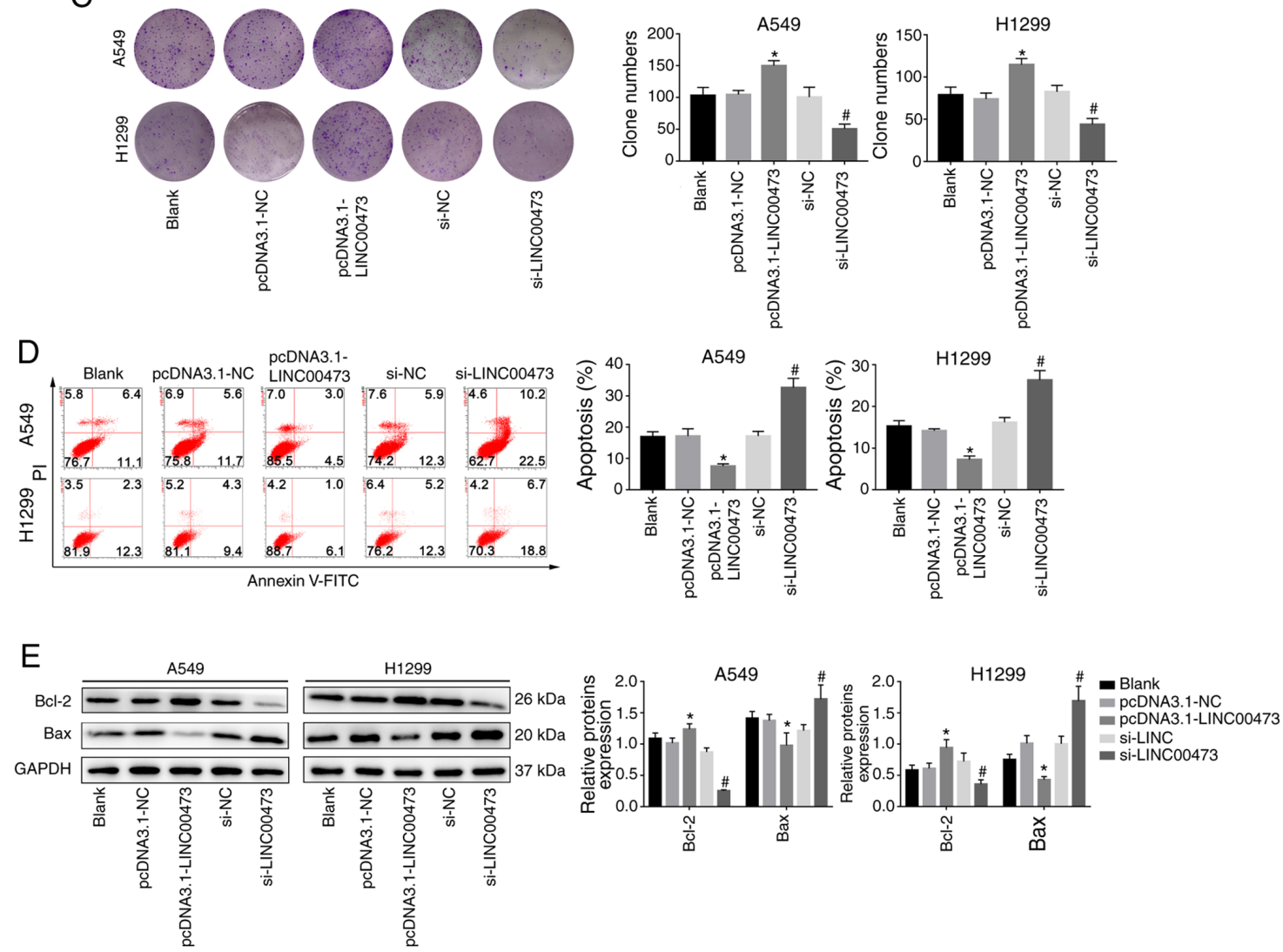

Figure 2. LINC00473 promotes the proliferation and inhibits apoptosis of NSCLC cell lines. (A) Expression of LINC00473 in NSCLC cell lines transfected with pc-DNA-LINC00473, si-LINC00473 and negative control. (B) MTT and (C) colony formation assays were used to detect the proliferation of NSCLC cells. (D) Apoptosis rate (\%) of A549 and H1299 cells. (E) Bcl-2 and Bax expression levels. ${ }^{*} \mathrm{P}<0.05$ vs. pcDNA3.1-NC and blank group; ${ }^{*} \mathrm{P}<0.05$ vs. si-NC and blank group. NSCLC, non-small cell lung cancer; NC, negative control; si, small interfering. 

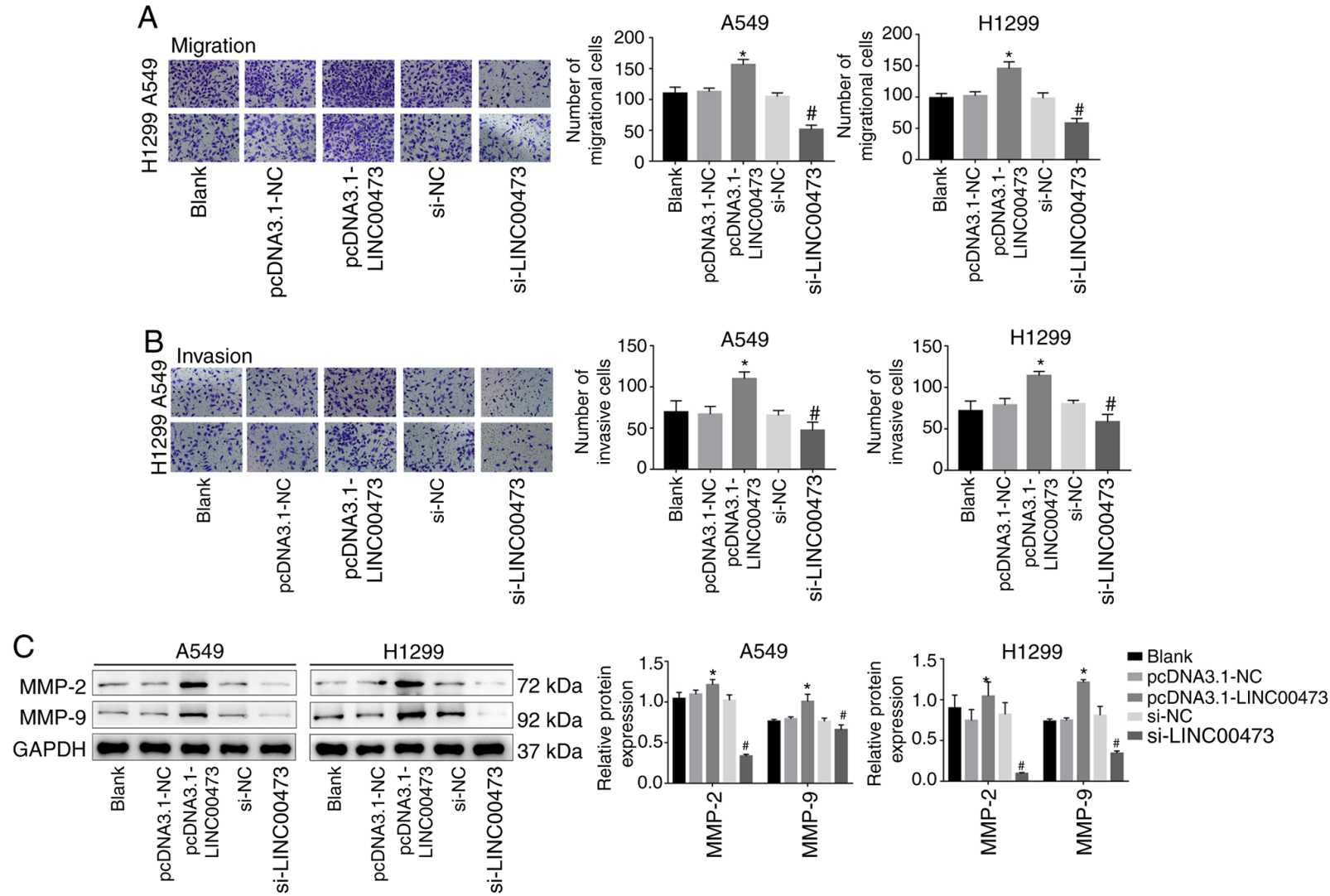

Figure 3. LINC00473 promotes migration and invasion of non-small cell lung cancer cell lines. (A) Migration and (B) invasion capacities of A549 and H1299 cells analysed using wound healing and Transwell migration assays, respectively (both magnification, x100). (C) Expression of MMP-2 and MMP-9 detected using western blotting. " $\mathrm{P}<0.05$ vs. pcDNA3.1-NC and blank group; " $\mathrm{P}<0.05$ vs. si-NC and blank group. NC, negative control; si, small interfering; MMP, matrix metalloproteinase.

LINC00473 competitively binds to miR-497-5p. As predicted by DIANA tools (http://carolina.imis.athena-innovation. gr/diana_tools/web/), miR-497-5p was a possible target miRNA of LINC00473. To confirm this, two types of luciferase reporter gene vectors (miR-497-5p-WT and miR-497-5p-MUT) were used to investigate whether LINC00473 acts as a sponge to miR-497-5p through direct binding (Fig. 4A). As shown in Fig. 4B and C, after transfection with WT sequences, the miR-497-5p mimics showed significantly inhibited luciferase activity (both $\mathrm{P}<0.05$ ). However, when the predicted binding site was mutated, overexpression of miR-497-5p showed no change in luciferase activity. Moreover, the expression of miR-497-5p was significantly reduced after overexpression of LINC00473 in both cell lines, but significantly increased after LINC00473-knockdown in A549 and H1299 cells (all $\mathrm{P}<0.05$; Fig. 4D and E). As shown in Fig. 4F, the expression of miR-497-5p in A549 and H1299 cells was lower compared with that in HBE (both $\mathrm{P}<0.05$ ). Likewise, the expression of miR-497-5p in 58 pairs of NSCLC and adjacent tissues was compared and it was reported that miR-497-5p was significantly downregulated in the former $(\mathrm{P}<0.05$; Fig. 4G). The expression of miR-497-5p was found to be negatively correlated with LINC00473 ( $\mathrm{P}<0.05$; Fig. 4H). The results indicated that LINC00473 could competitively bind to miR-497-5p and negatively regulate its expression.

miR-497-5p inhibition attenuates the effect of LINC00473 silencing. As depicted in Fig. 5A, the cell proliferation rates in the si-LINC00473 group were lower compared with the NC group. However, cell viability was enhanced after treatment with miR-497-5p inhibitors $(\mathrm{P}<0.05)$. The clone number of A549 cells decreased after LINC00473-knockdown but increased after the addition of miR-497-5p inhibitors ( $\mathrm{P}<0.05$; Fig. 5B). Moreover, miR-497-5p inhibition stabilised the increase in apoptosis and the expression of apoptosis-related proteins induced by LINC00473-knockdown (all P<0.05; Fig. 5C and D). Transwell migration assays indicated that both cell migration and invasion abilities were reduced after silencing LINC00473 but were reversed after co-transfection with miR-497 inhibitors (both $\mathrm{P}<0.05$; Fig. 6A). Similarly, the expression of MMP-2 and MMP-9 was inhibited by silencing LINC00473 but was reversed by miR-497 inhibitors (all $\mathrm{P}<0.05$; Fig. 6B). The current results suggested that LINC00473 served its role by regulating miR-497-5p expression.

LINC00473 activates the MAPK signalling pathway. To study the relationship between LINC00473 and the MAPK signalling pathway, the expression of proteins associated with the pathway was analysed. The data indicated that p-ERK1/2 and p-p38 expression increased when LINC00473 was overexpressed and decreased when LINC00473 was knocked down (Fig. 7A). Moreover, the p-ERK1/2/ERK1/2 ratio $(\mathrm{P}<0.05$; Fig. $7 \mathrm{~B})$ and $\mathrm{p}-\mathrm{p} 38 / \mathrm{p} 38$ ratio showed the similar trends. In A459 cells, the expression levels of p-ERK1/2 and p-p38 were significantly decreased by LINC00473-knockdown and increased after treatment with miR-497-5p inhibitors (and 


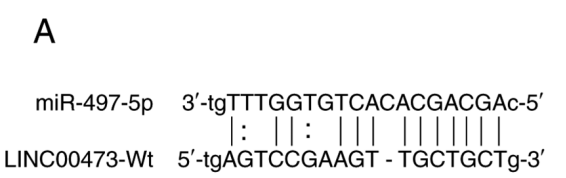

B

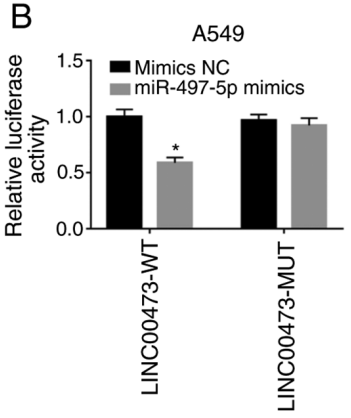

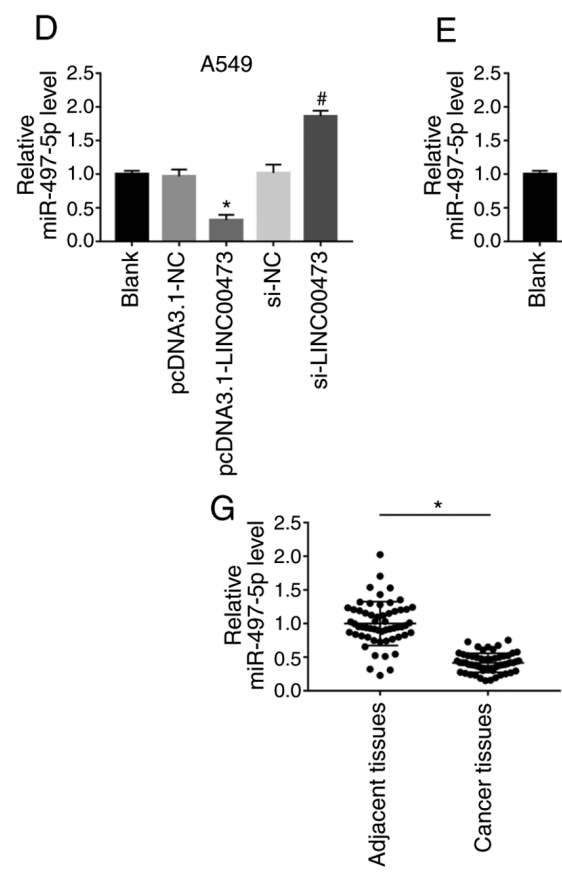

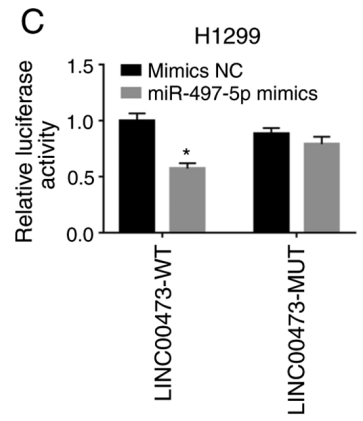

$\mathrm{F}$
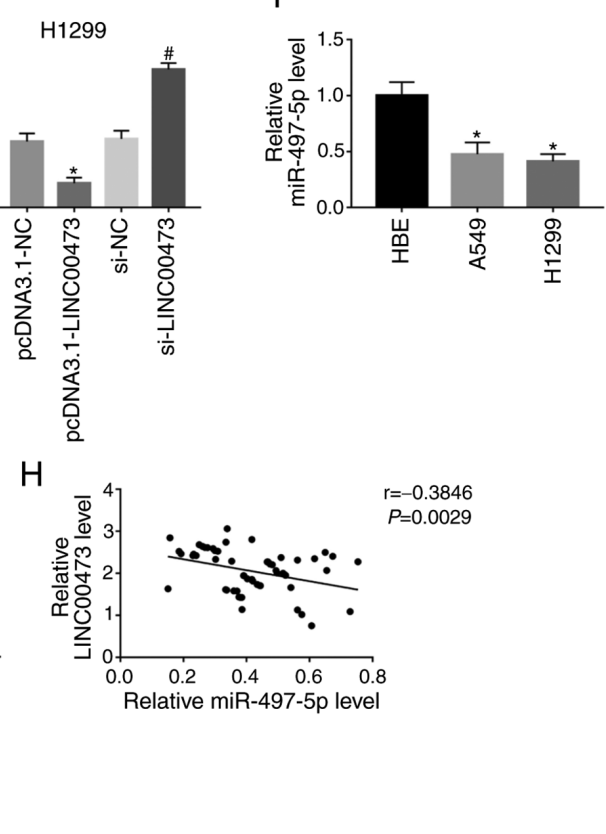

Figure 4. LINC00473 targets miR-497-5p. (A) Binding site of LINC00473 and miR-497-5p predicted using DIANA. Relative activity of luciferase activity in the (B) A549 and (C) H1299 cell lines. Expression of miR-497-5p in (D) A549 and (E) H1299 cells transfected with pcDNA-LINC00473 and si-LINC00473. (F) Expression of miR-497-5p in HBE and the NSCLC cell lines A549 and H1299. (G) miR-497-5p expression in 58 pairs of lung cancer-afflicted and adjacent tissues. (H) Correlation analysis of LINC00473 and miR-497-5p in lung cancer. " $\mathrm{P}<0.05$ vs. pcDNA3.1-NC, blank group or HBE cells; ${ }^{*} \mathrm{P}<0.05$ vs. si-NC and blank group. si, small interfering; miR, microRNA; HBE, human bronchial epithelial; Wt, wild-type.

both p-ERK1/2/ERK1/2 ratio and p-p38/p38 ratio showed the same trends $(\mathrm{P}<0.05$; Fig. $7 \mathrm{C}$ and $\mathrm{D})$. These results indicated that the regulatory mechanism of LINC00473 may be through the MAPK signalling pathway.

\section{Discussion}

Multiple studies have suggested that IncRNAs play crucial roles in tumour progression, making them promising therapeutic targets for treatment of diseases such as NSCLC (16-18). Some studies have reported that the lncRNA LINC00473 promotes cell migration and invasion in numerous types of cancer, including pancreatic, mucoepidermoid and breast cancer $(11,19,20)$. He (21) reported that LINC00473 regulates the progression of oesophageal squamous cell carcinoma by affecting 5'-AMP-activated protein kinase catalytic subunit $\alpha-1$ expression. Furthermore, Mo et al (22) revealed that LINC00473 promotes the progression of hepatocellular carcinoma by sponging miRNA-195 and increasing high mobility group protein HMGI-C expression. However, whether miR-146-5p is involved in the regulation of lung cancer remains unclear. The present study demonstrated that LINC00473 was significantly upregulated in both patient lung cancer tissues and NSCLC cell lines. Additionally, the inhibitory effects of silencing LINC00473 and the promotional effect of its overexpression on the proliferation, invasion and migration of NSCLC cells was investigated.

Several studies have suggested that lncRNAs act as natural sponges to interact with miRNAs by eliminating the inhibitory activity of these miRNAs (23-25). Chen et al (24) showed that LINC00473 weakens the effect of radiotherapy by targeting miR-374a-5p. Wang et al (25) also revealed that in colorectal cancer LINC00473 can promote Taxol resistance by inhibiting miR-15a. In the present study, to explore the underlying mechanism of LINC00473 in NSCLC development, target miRNAs of LINC00473 were investigated. Using bioinformatics methods, it was demonstrated that miR-497-5p contained a binding site for LINC00473. Luciferase reporter assays confirmed that LINC00473 and miR-497-5p bind with each other. Furthermore, the expression of miR-497-5p was decreased by LINC00473-overexpression and enhanced by LINC00473 silencing. Moreover, the inhibition of miR-497-5p attenuated the effect of LINC00473 silencing on cell proliferation, apoptosis, migration and invasion. These data 


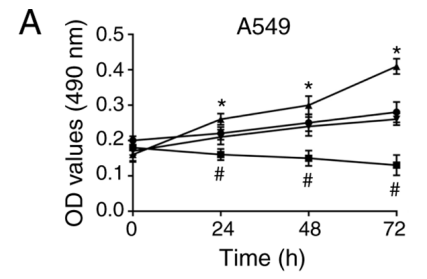

$\rightarrow$ si-NC+inhibitors NC

- si-LINC00473+inhibitors NC

+ si-NC+miR-497-5p inhibitors

* si-LINC00473+miR-497-5p inhibitors
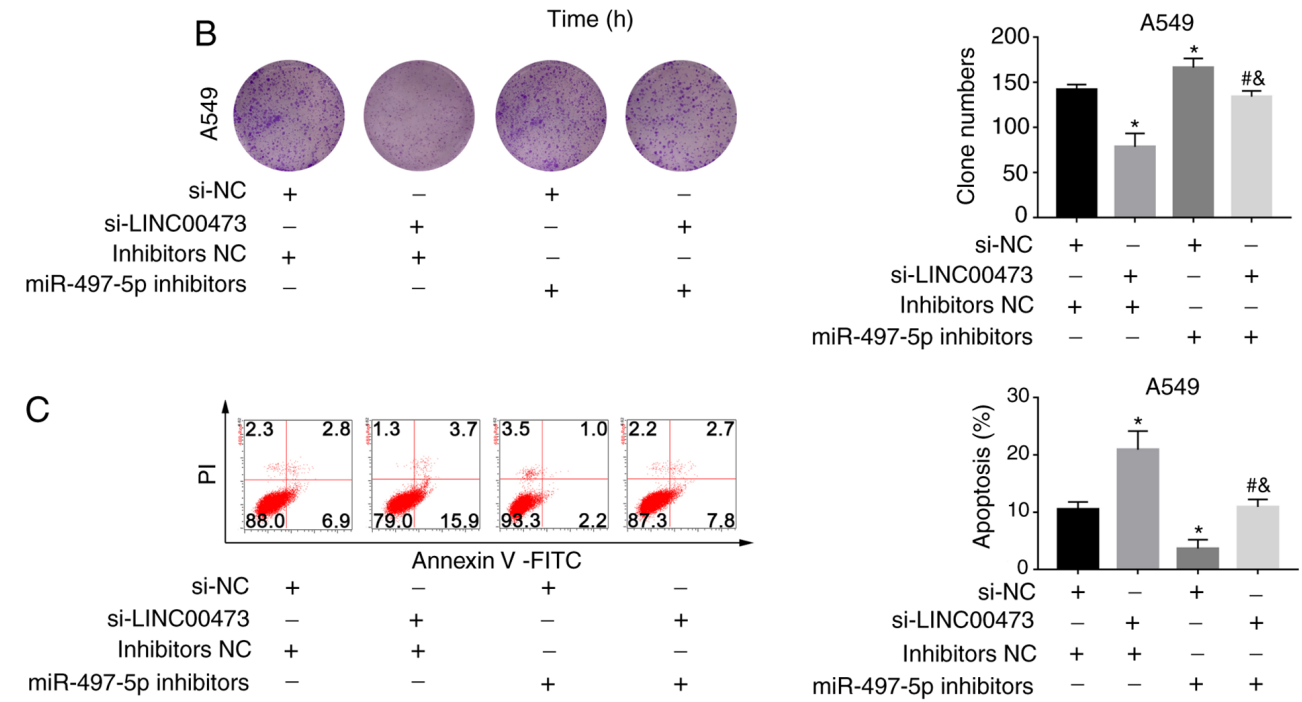

C
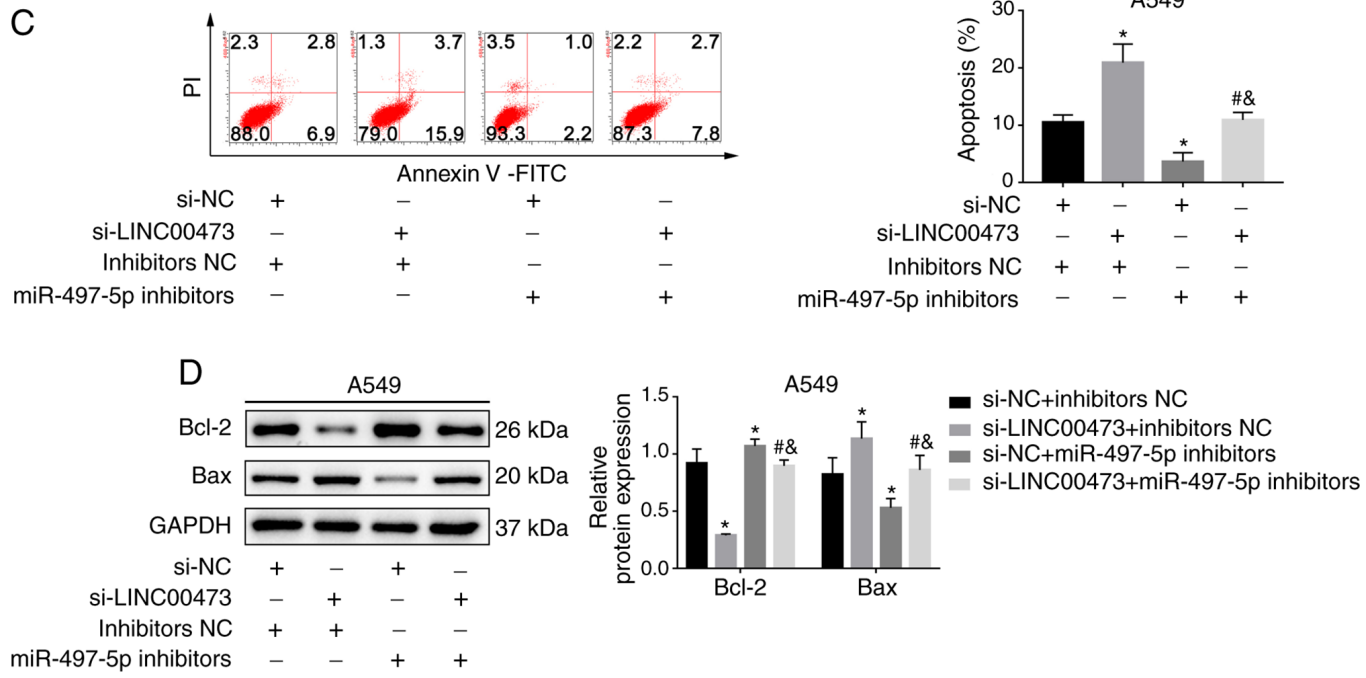

Figure 5. miR-497-5p reverses the effect of LINC00473 on the proliferation and apoptosis of non-small cell lung cancer cells. (A and B) Proliferation ability and (C) analysis of apoptosis rate (\%) and (D) Bcl-2 and Bax expression in A549 cells.
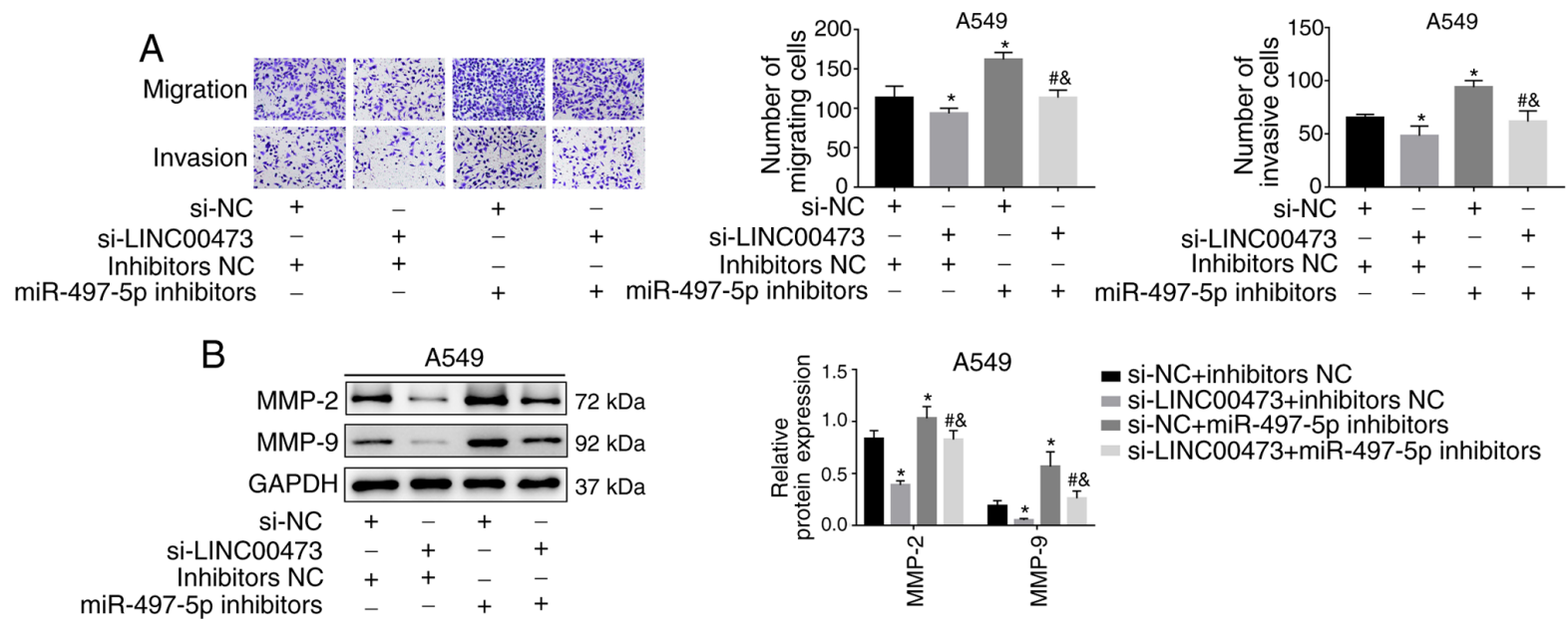

Figure 6. miR-497-5p reverses the effect of LINC00473 on the migration of non-small cell lung cancer cells. (A) Analysis of migration and invasion capacity of A549 cells (magnification, x10). (B) Expression of MMP-2 and MMP-9. ${ }^{*} \mathrm{P}<0.05$ vs. si-NC + inhibitors NC group; ${ }^{~} \mathrm{P}<0.05$ vs. si-LINC00473 + inhibitors $\mathrm{NC}$ group; ${ }^{\circledR} \mathrm{P}<0.05$ vs. si-NC + miR-497-5p inhibitors group. MMP, matrix metalloproteinase; NC, negative control; si, small interfering.

suggested that LINC00473 promotes NSCLC progression in an miR-497-5p-dependent manner.

It has been reported that the MEK/ERK signalling pathway is involved in cell proliferation, apoptosis and migration (26). Several lncRNAs, such as lncRNA-01126 and lncRNA HOTTIP, have been shown to exert their functions by regulating this pathway $(27,28)$. This pathway is involved in the migration and invasion of NSCLC cells induced by the 

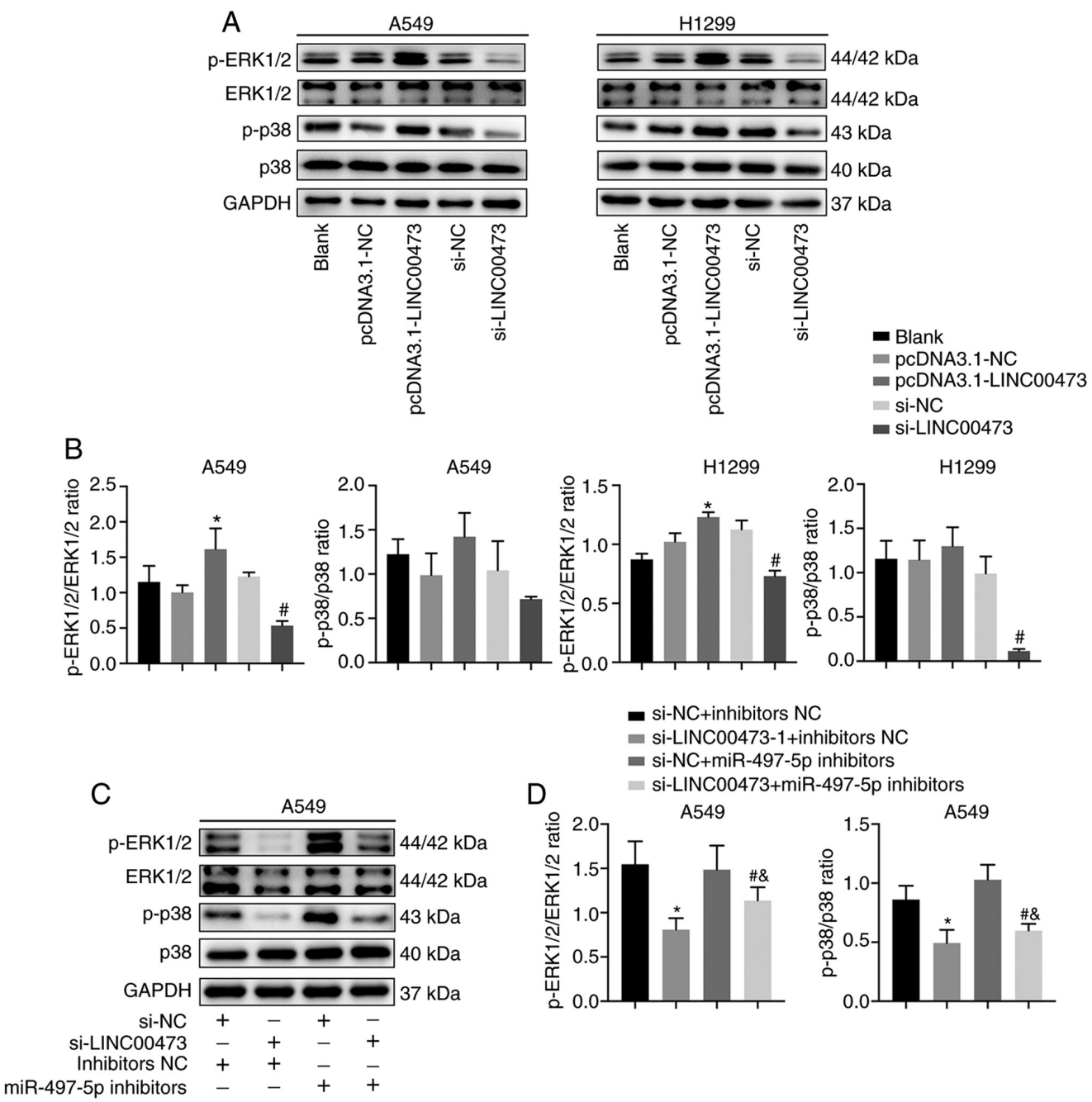

Figure 7. LINC00473 activates the MAPK signalling pathway. (A) Expression of MAPK-related proteins in non-small cell lung cancer cells treated with pcDNA3.1-LINC00473 or si-LINC00473. (B) Ratio of p-ERK1/2 to ERK1/2 and p-p38 to p38. " $\mathrm{P}<0.05$ vs. pcDNA3.1-NC; ${ }^{*} \mathrm{P}<0.05$ vs. si-NC. (C) Expression of MAPK-related proteins in A549 cells transfected with si-LINC00473 or miR-497-5p inhibitors. (D) Ratio of p-ERK1/2 to ERK1/2 and p-p38 to p38. "P $<0.05$ vs. si-NC + inhibitors NC group; ${ }^{\#} \mathrm{P}<0.05$ vs. si-LINC00473 + inhibitors NC group; ${ }^{\text {\& }} \mathrm{P}<0.05$ vs. si-NC + miR-497-5p inhibitors group.

lncRNA SDPR-AS (29). In addition, the IncRNA MALAT1 has been shown to play a tumour-enhancing role by regulating the development of NSCLC via the MAPK/ERK pathway (30). The present results indicated that LINC00473-knockdown decreased the p-ERK and p-p38 levels, which were reversed by miR-497-5p inhibition. These data indicated that LINC00473 regulates the activity of the MAPK/ERK signalling pathway to influence the proliferation, migration and invasion of NSCLC cells.

Overall, the present results indicated that LINC00473 is highly expressed in NSCLC tissues, and its downregulation suppresses the malignant activities of NSCLC cells. Structurally, LINC00473 competitively binds with miR-497-5p and regulates the MEK/ERK signalling pathway.

\section{Acknowledgements}

Not applicable.

\section{Funding}

No funding was received.

\section{Availability of data and materials}

The datasets used and analysed during the current study are available from the corresponding author on reasonable request.

\section{Authors' contributions}

MJS conceived, interpreted the data and designed the study. MJS and SHX confirmed the authenticity of all the raw data. SHX, YHB, HCM and HNZ performed the experiments and analysed the data. SHX wrote the manuscript. All authors read and approved the final manuscript.

\section{Ethics approval and consent to participate}

The present study was approved by The Ethics Committee of The Second Hospital of Shandong University (Ji'nan, China) and all patients provided written informed consent.

\section{Patient consent for publication}

Not applicable. 


\section{Competing interests}

The authors declare that they have no competing interests.

\section{References}

1. Siegel RL, Miller KD and Jemal A: Cancer statistics, 2019. CA Cancer J Clin 69: 7-34, 2019.

2. Inamura K: Lung cancer: Understanding its molecular pathology and the 2015 WHO classification. Front Oncol 7: 193, 2017.

3. Bray F, Ferlay J, Soerjomataram I, Siegel RL, Torre LA and Jemal A: Global cancer statistics 2018: GLOBOCAN estimates of incidence and mortality worldwide for 36 cancers in 185 countries. CA Cancer J Clin 68: 394-424, 2018.

4. Laskin JJ and Sandler AB: State of the art in therapy for non-small cell lung cancer. Cancer Invest 23: 427-442, 2005.

5. Choi SW, Kim HW and Nam JW: The small peptide world in long noncoding RNAs. Brief Bioinform 20: 1853-1864, 2019.

6. Chekulaeva M and Rajewsky N: Roles of long noncoding RNAs and circular RNAs in translation. Cold Spring Harb Perspect Biol 11: a032680, 2019.

7. Li M, Chen Y, Zhu J, Gao Z, Wang T and Zhou P: Long noncoding RNA CASC15 predicts unfavorable prognosis and exerts oncogenic functions in non-small cell lung cancer. Am J Transl Res 11: 4303-4314, 2019.

8. Sun Z, He C, Xiao M, Wei B, Zhu Y, Zhang G, Zhou H, Yuan J, $\mathrm{Hu} \mathrm{X}$ and Yi Y: LncRNA FOXC2 antisense transcript accelerates non-small-cell lung cancer tumorigenesis via silencing p15. Am J Transl Res 11: 4552-4560, 2019.

9. Yao G, Chen K, Qin Y, Niu Y, Zhang X, Xu S, Zhang C, Feng M and Wang K: Long non-coding RNA JHDM1D-AS1 interacts with DHX15 protein to enhance non-small-cell lung cancer growth and metastasis. Mol Ther Nucleic Acids 18: 831-840, 2019.

10. Bhan A, Soleimani M and Mandal SS: Long noncoding RNA and cancer: A new paradigm. Cancer Res 77: 3965-3981, 2017.

11. Zhou WY, Zhang MM, Liu C, Kang Y, Wang JO and Yang XH: Long noncoding RNA LINC00473 drives the progression of pancreatic cancer via upregulating programmed death-ligand 1 by sponging microRNA-195-5p. J Cell Physiol 234: 23176-23189, 2019.

12. Niu L, Zhou Y, Zhang W and Ren Y: Long noncoding RNA LINC00473 functions as a competing endogenous RNA to regulate MAPK1 expression by sponging miR-198 in breast cancer. Pathol Res Pract 215: 152470, 2019.

13. Stavast CJ and Erkeland SJ: The non-canonical aspects of MicroRNAs: Many roads to gene regulation. Cells 8: E1465, 2019.

14. Paraskevopoulou MD and Hatzigeorgiou AG: Analyzing MiRNA-LncRNA interactions. Methods Mol Biol 1402: 271-286, 2016.

15. Livak KJ and Schmittgen TD: Analysis of relative gene expression data using real-time quantitative PCR and the $2(-\Delta \Delta \mathrm{C}(\mathrm{T}))$ method. Methods 25: 402-408, 2001.

16. Lu T, Wang Y, Chen D, Liu J and Jiao W: Potential clinical application of lncRNAs in non-small cell lung cancer. OncoTargets Ther 11: 8045-8052, 2018.

17. Zhou Y, Shi H, Du Y, Zhao G, Wang X, Li Q, Liu J, Ye L, Shen Z, Guo Y, et al: 1ncRNA DLEU2 modulates cell proliferation and invasion of non-small cell lung cancer by regulating miR-30c-5p/SOX9 axis. Aging (Albany NY) 11: 7386-7401, 2019.
18. Shi J, Li J, Yang S, Hu X, Chen J, Feng J, Shi T, He Y, Mei Z, He W, et al: LncRNA SNHG3 is activated by E2F1 and promotes proliferation and migration of non-small-cell lung cancer cells through activating TGF- $\beta$ pathway and IL-6/JAK2/STAT3 pathway. J Cell Physiol 235: 2891-2900, 2020.

19. Chen Z, Lin S, Li J-L, Ni W, Guo R, Lu J, Kaye FJ and Wu L: CRTC1-MAML2 fusion-induced 1ncRNA LINC00473 expression maintains the growth and survival of human mucoepidermoid carcinoma cells. Oncogene 37: 1885-1895, 2018.

20. Shi X and Wang X: LINC00473 mediates cyclin D1 expression through a balance between activation and repression signals in breast cancer cells. FEBS Lett 593: 751-759, 2019.

21. He Z: LINC00473/miR-497-5p regulates esophageal squamous cell carcinoma progression through targeting PRKAA1. Cancer Biother Radiopharm 34: 650-659, 2019.

22. Mo J, Li B, Zhou Y, Xu Y, Jiang H, Cheng X, Wu X and Zhang Y: LINC00473 promotes hepatocellular carcinoma progression via acting as a ceRNA for microRNA-195 and increasing HMGA2 expression. Biomed Pharmacother 120: 109403, 2019.

23. Li D, Li H, Yang Y and Kang L: Long Noncoding RNA urothelial carcinoma-associated 1 promotes the proliferation and metastasis of human lung tumor cells by regulating MicroRNA-144. Oncol Res 26: 537-546, 2018.

24. Chen W, Zhang Y, Wang H, Pan T, Zhang Y and Li C: LINC00473/ miR-374a-5p regulates esophageal squamous cell carcinoma via targeting SPIN1 to weaken the effect of radiotherapy. J Cell Biochem 120: 14562-14572, 2019.

25. Wang L,Zhang X, Sheng L, Qiu C and Luo R: LINC00473 promotes the Taxol resistance via miR-15a in colorectal cancer. Biosci Rep: Sep 20,2018 (Epub ahead of print). doi: 10.1042/BSR20180790.

26. Sun Y, Liu W-Z, Liu T, Feng X, Yang N and Zhou H-F: Signaling pathway of MAPK/ERK in cell proliferation, differentiation, migration, senescence and apoptosis. J Recept Signal Transduct Res 35: 600-604, 2015.

27. Zhu Y, Ai R, Ding Z, He Q, Zhang X, Dong Y and He Y: LncRNA-01126 inhibits the migration of human periodontal ligament cells through MEK/ERK signaling pathway. J Periodontal Res 55: 631-641, 2020

28. Liu J, Hu HB, Liu YM, Li FX, Zhang LP and Liao ZM: LncRNA HOTTIP promotes the proliferation and invasion of ovarian cancer cells by activating the MEK/ERK pathway. Mol Med Rep 22: 3667-3676, 2020.

29. Zhu J, Wang L and Liao R: Long non-coding RNA SDPR-AS affects the development of non-small cell lung cancer by regulating SDPR through p38 MAPK/ERK signals. Artif Cells Nanomed Biotechnol 47: 3172-3179, 2019.

30. Liu C,LiH, Jia J, Ruan X,Liu Y and Zhang X:High metastasis-associated lung adenocarcinoma transcript 1 (MALAT1) expression promotes proliferation, migration, and invasion of non-small cell lung cancer via ERK/mitogen-activated protein kinase (MAPK) signaling pathway. Med Sci Monit 25: 5143-5149, 2019.

This work is licensed under a Creative Commons Attribution-NonCommercial-NoDerivatives 4.0 International (CC BY-NC-ND 4.0) License. 